\title{
Disruption of mitochondrial fission in the liver protects mice from diet-induced obesity and metabolic deterioration
}

\author{
Lixiang Wang ${ }^{1}$. Takaya Ishihara ${ }^{2}$ - Yuta Ibayashi ${ }^{1}$ Keita Tatsushima ${ }^{1}$. \\ Daiki Setoyama $^{3}$ - Yuki Hanada ${ }^{1}$. Yukina Takeichi ${ }^{1}$ - Shohei Sakamoto ${ }^{1}$. \\ Sadaki Yokota $^{4}$ - Katsuyoshi Mihara ${ }^{5}$ - Dongchon Kang ${ }^{3}$ - Naotada Ishihara ${ }^{2}$. \\ Ryoichi Takayanagi ${ }^{1} \cdot$ Masatoshi Nomura $^{1}$
}

Received: 5 April 2015 / Accepted: 1 July 2015 /Published online: 2 August 2015

(C) Springer-Verlag Berlin Heidelberg 2015

\begin{abstract}
Aim/hypothesis Mitochondria and the endoplasmic reticulum (ER) physically interact by close structural juxtaposition, via the mitochondria-associated ER membrane. Inter-organelle communication between the ER and mitochondria has been shown to regulate energy metabolism and to be central to the modulation of various key processes such as ER stress. We aimed to clarify the role of mitochondrial fission in this communication.

Methods We generated mice lacking the mitochondrial fission protein dynamin-related protein 1 (DRP1) in the liver (DrplLiKO mice).

Results Drp1LiKO mice showed decreased fat mass and were protected from high-fat diet (HFD)-induced obesity. Analysis of liver gene expression profiles demonstrated marked elevation of ER stress markers. In addition, we observed increased
\end{abstract}

Electronic supplementary material The online version of this article (doi:10.1007/s00125-015-3704-7) contains peer-reviewed but unedited supplementary material, which is available to authorised users.

Masatoshi Nomura

nomura@med.kyushu-u.ac.jp

1 Department of Medicine and Bioregulatory Science, Graduate School of Medical Science, Kyushu University, Maidashi 3-1-1, Higashi-ku, Fukuoka 812-8582, Japan

2 Department of Protein Biochemistry, Institute of Life Science, Kurume University, Kurume, Japan

3 Department of Clinical Chemistry and Laboratory Medicine, Graduate School of Medical Science, Kyushu University, Fukuoka, Japan

4 Division of Functional Morphology, Faculty of Pharmaceutical Sciences, Nagasaki International University, Sasebo, Nagasaki, Japan

5 Department of Molecular Biology, Graduate School of Medical Science, Kyushu University, Fukuoka, Japan expression of the fibroblast growth factor 21 (FGF21) gene through induction of activating transcription factor 4 , master regulator of the integrated stress response.

Conclusions/interpretation Disruption of mitochondrial fission in the liver provoked ER stress, while inducing the expression of FGF21 to increase energy expenditure and protect against HFD-induced obesity.

Keywords DRP1 · ER stress · FGF $21 \cdot$ Mitochondria-ER juxtaposition structure $\cdot$ Mitochondrial dynamics

$\begin{array}{ll}\text { Abbreviations } \\ \text { ALT } & \text { Alanine aminotransferase } \\ \text { AST } & \text { Aspartate aminotransferase } \\ \text { ATF4 } & \text { Activating transcription factor } 4 \\ \text { BAT } & \text { Brown adipose tissue } \\ \text { CHOP } & \text { C/EBP homologous protein } \\ \text { DRP1 } & \text { Dynamin-related protein 1 } \\ \text { Drp1LiKO } & \text { Liver-specific Drp1 knockout } \\ \text { eIF2 } \alpha & \text { Eukaryotic translation factor } 2 \alpha \\ \text { ER } & \text { Endoplasmic reticulum } \\ \text { FGF21 } & \text { Fibroblast growth factor } 21 \\ \text { HFD } & \text { High-fat diet } \\ \text { IPGTT } & \text { Intraperitoneal glucose tolerance test } \\ \text { IRE1 } \alpha & \text { Inositol-requiring } 1 \alpha \\ \text { ITT } & \text { Insulin tolerance test } \\ \text { MAM } & \text { Mitochondria-associated ER membrane } \\ \text { MFF } & \text { Mitochondrial fission factor } \\ \text { MFN } & \text { Mitofusin } \\ \text { NCD } & \text { Normal chow diet } \\ \text { PERK } & \text { Protein kinase R-like ER kinase } \\ \text { RER } & \text { Respiratory exchange ratio } \\ \text { WAT } & \text { White adipose tissue }\end{array}$




\section{Introduction}

Mitochondria are highly dynamic organelles that frequently fuse and divide in response to cellular energy demands, differentiation or pathological conditions [1-3]. The endoplasmic reticulum (ER) is an extensive, morphologically continuous network of membrane tubules in the eukaryotic cell. It performs a variety of functions in cells including lipid synthesis, intracellular $\mathrm{Ca}^{2+}$ regulation and protein secretion [4]. Recently, it was reported that the ER plays an active role in defining the sites of mitochondrial division [5]. In fact, the two organelles physically interact, forming specialised contacts referred to as mitochondria-associated ER membrane (MAM). MAM has been linked with lipid transfer, apoptotic calcium signalling and mitochondria-ER calcium homeostasis [6]. Furthermore, MAM has recently been shown to regulate mitochondrial shape and motility, energy metabolism and redox status and to be central to the modulation of various key processes such as ER stress, autophagy and inflammasome signalling [7].

In vertebrates, mitofusin-1 and -2 (MFN1 and MFN2) are involved in mitochondrial fusion and dynamin-related protein 1 (DRP1) and mitochondrial fission factor (MFF) control mitochondrial fission $[8,9]$. During the fission cycle, DRP1 first binds to MFF on the surface of mitochondria, followed by entry into a complex that includes ER proteins at the ER-mitochondria interface [10]. Reciprocally, ER-localised inverted formin 2-mediated actin polymerisation is required for efficient mitochondrial fission [11], suggesting that ER contact is required for initiation of mitochondrial fission. Recently, MFN2 has been shown to be involved in MAM architecture and to regulate insulin signalling and insulin sensitivity in muscle and liver [12]. Furthermore, MAM integrity is required for insulin signalling and is implicated in hepatic insulin resistance [13]. These results led us to hypothesise that DRP1 plays a role in glucose homeostasis through organelle communication between the ER and mitochondria. To investigate such a role of mitochondrial fission, we generated mice with liver-specific deletion of Drpl (DrplLiKO). DrplLiKO mice showed decreased fat mass and were protected from high-fat diet (HFD)-induced obesity. Further analysis revealed that defects of mitochondrial fission in the liver cause ER stress, leading to induction of fibroblast growth factor 21 (FGF21), a metabolic regulator of glucose and lipid homeostasis. In this study, we show that mitochondrial fission in the liver plays an important role in energy homeostasis through induction of FGF21.

\section{Methods}

Animal experiments DrplLiKO mice were generated by crossing Drp $1^{\text {flox/+ }}$ mice [14] with Alb-Cre mice. Mice were fed ad libitum with a normal chow diet (NCD) (5.4\% fat, CRF-1; Orient Yeast, Tokyo, Japan) and kept under a $12 \mathrm{~h}$ light-dark cycle. For the HFD study, 4-week-old mice were put on an HFD (24\% fat, lard fat, $188.28 \mathrm{~kJ} \%$ fat, D12451; Research Diets, New Brunswick, NJ, USA) for 16-24 weeks. See electronic supplementary material (ESM) Methods for further details.

Western blot The in vivo insulin sensitivity was assessed by quantification of Akt phosphorylation in response to exogenous insulin. See ESM Methods for details.

Microscopic analyses For histochemical and immunohistochemical analyses, adipose tissue and livers were fixed, processed, stained and quantified. The morphologies of mitochondria and ER were analysed by electron microscopy. See ESM Methods for details.

mRNA analyses Real-time quantitative RT-PCR was used to determine the relative expression levels of mRNA. Results were normalised to Gapdh expression. Liver mRNA from control and DrplLiKO mice was subjected to microarray analysis. See ESM Methods for details.

Biochemical assays Total liver lipid content was determined by the Folch method [15]. For VLDL secretion assays, mice were fasted for $4 \mathrm{~h}$ before being injected with $0.5 \mathrm{~g} / \mathrm{kg}$ tyloxapol (Triton-1339; Sigma, St Louis, MO, USA). See ESM Methods for details.

Metabolic studies Body weight, food intake and locomotor activity were measured. Glucose and insulin tolerance tests were performed as described [16]. See ESM Methods for details.

Oxygen consumption The rate of oxygen consumption of mitochondria was measured using an oxygen electrode as described [14]. See ESM Methods for details.

Statistical analysis All data are expressed as means $\pm \mathrm{SE}$ and evaluated by Student's $t$ test using Microsoft Excel software (Mac 2011 version 14.3.5; Microsoft Japan, Tokyo, Japan), two-way ANOVA performed with Tukey's post hoc test or repeated measures two-way ANOVA performed with the Bonferroni post hoc test using GraphPad Prism 6.0 software (GraphPad; San Diego, CA, USA). To detect an outlier, we performed the Grubbs' test using the free statistical calculators: QuickCals, http://www.graphpad.com/quickcalcs/. Significance levels were set at $p<0.05, p<0.01$ and $p<0.001$. 


\section{Results}

Ablation of Drp1 protects mice from HFD-induced obesity As shown in Fig. 1a, DRP1 protein was ablated in the liver of Drp ILiKO mice. RT-PCR revealed approximately $80 \%$ reduction of Drp1 mRNA levels in the liver of DrplLiKO

a
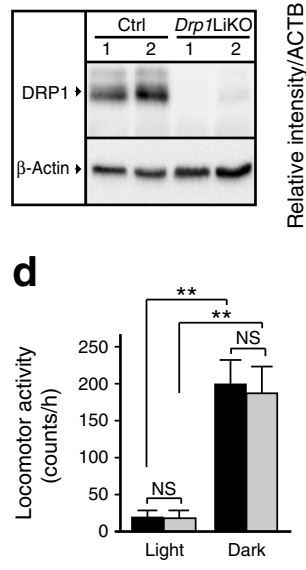

b

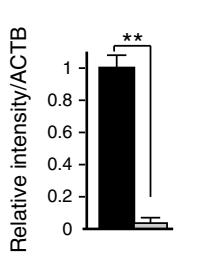

e

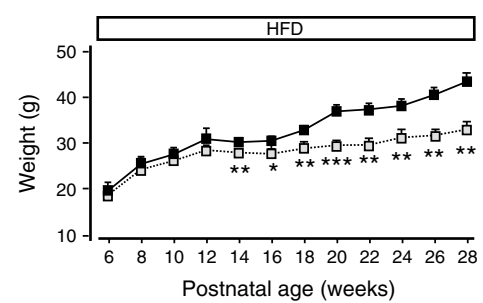

f

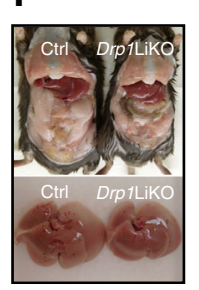

g

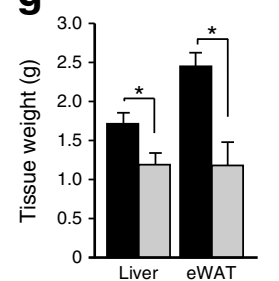

j h

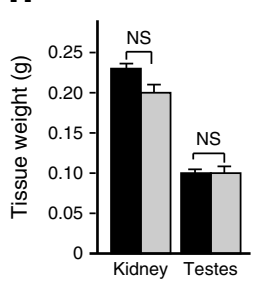

$\mathbf{k}$

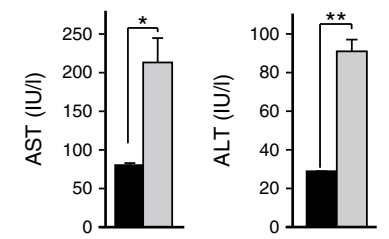

i

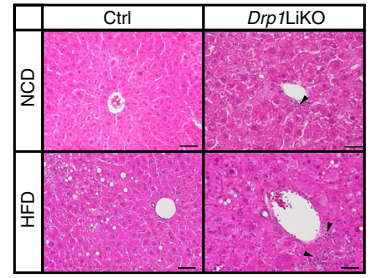

Fig. 1 Ablation of Drpl protects mice from HFD-induced obesity. (a) Immunoblot analyses of DRP1 protein from the livers of two control and two DrplLiKO mice. The bar graph shows the intensities of protein bands quantified by densitometric scanning. (b) Drpl expression levels determined by real-time quantitative RT-PCR analysis. (c) Food intake in 24-week-old HFD-fed Drp1LiKO and control mice $(n=5)$. (d) Locomotor activity in 18-week-old HFD-fed Drp 1 LiKO and control mice $(n=5)$. (e) Body weight of DrplLiKO (grey squares, $n=8$ ) and control mice (black squares, $n=8$ ) fed an HFD from 6 weeks of age. (f) Representative image of 24-week-old HFD-fed DrplLiKO and control mice. (g, h) Tissue weights of 24-week-old HFD-fed DrplLiKO and control mice: liver and epididymal WAT (eWAT) (g, $n=5)$; kidney and testes (h, $n=5)$. (i) Haematoxylin-eosin staining of livers from 20 -week-old control and DrplLiKO mice on either NCD or HFD. Arrows indicate inflammatory cell infiltration. Scale bar, $50 \mu \mathrm{m}$. (j, k) Serum AST (j) and ALT (k) values in control and DrplLiKO mice $(n=5)$. Black bars, control mice; grey bars, Drp 1 LiKO mice. Values are expressed as means $\pm \mathrm{SE}$. ${ }^{*} p<0.05,{ }^{* *} p<0.01$ and ${ }^{* * *} p<0.001$, as calculated by Student's $t$ test $(\mathbf{a}-\mathbf{c}, \mathbf{e}, \mathbf{g}, \mathbf{h}, \mathbf{j}, \mathbf{k})$ or two-way ANOVA (d)
(Fig. 1b). On the NCD, no apparent difference was found in body weight of the DrplLiKO mice compared with controls (ESM Fig. 1a). In striking contrast, when fed an HFD, although the food intake and locomotor activity were nearly identical (Fig. 1c, d), the body weight of DrplLiKO mice was significantly lower than that of control mice (Fig. 1e). Liver and epididymal white adipose tissue (WAT) weights were decreased in Drp/LiKO mice (Fig. 1f, g), while there were no significant differences in the weight of other tissues such as kidney and testes (Fig. 1h). Collectively, DrplLiKO mice showed decreased fat mass and were protected from HFD-induced obesity. When fed an HFD, hematoxylin-eosin staining of liver sections revealed that disorganised lobular parenchyma displayed inflammatory cell infiltration in Drp ILiKO mice (Fig. 1i). The inflammatory cells were identified as small cells with little cytoplasm and formed a cluster.

When fed an NCD, reduced inflammatory cell infiltration was found in DrplLiKO mice (Fig. 1i). Reflecting hepatic inflammation, serum aspartate aminotransferase (AST) and alanine aminotransferase (ALT) levels were 3.17-fold and 2.93-fold higher in the HFD DrplLiKO mice, respectively, when compared with control mice (Fig. 1j, k).

Hepatic lipid content in Drp1LiKO mice is comparable with that in control mice We analysed the hepatic lipid content and the expression of the genes involved in lipid metabolism in the livers of HFD-fed mice. There were no significant differences in triacylglycerol, total cholesterol, total NEFA or total lipid contents in the livers of control and Drp/LiKO mice (Fig. 2a-d). The mRNA levels of the genes involved in lipogenesis and $\beta$-oxidation were analysed. The gene encoding sterol regulatory element binding transcription factor 1 (Srebp1c, also known as Srebfl) was expressed at lower levels in DrplLiKO mice than in controls in both the fasting and refed condition (Fig. 2e), whereas expression levels of the genes encoding fatty acid synthetase (Fasn), acetyl-CoA carboxylase $(A c c)$ and carnitine palmitoyltransferase Ia (Cptla) were no different (Fig. 2f-h). Collectively, lipogenesis was slightly impaired, while lipolysis was not affected in the liver of DrplLiKO mice on an HFD.

Decreased hepatic VLDL secretion in Drp1LiKO mice The levels of serum triacylglycerol and total cholesterol were significantly lower in DrplLiKO mice than in control mice after $17 \mathrm{~h}$ fasting, while the total serum NEFA level was no different (Fig. 2i-k). Since food intake increases hepatic fatty acid uptake and VLDL secretion, these lipid profiles were also analysed after refeeding for $4 \mathrm{~h}$. The levels of serum triacylglycerol and total cholesterol were significantly lower in DrplLiKO mice than in control mice. It was noted that the increase in serum triacylglycerol after feeding was completely negated in DrplLiKO mice (Fig. 2i), suggesting that the defect may also reside in VLDL secretion in DrplLiKO mice. 


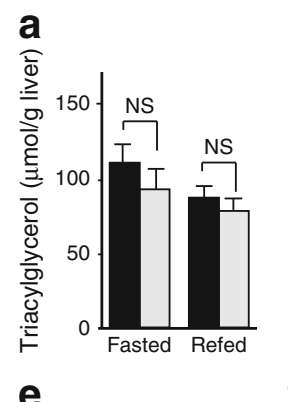

b

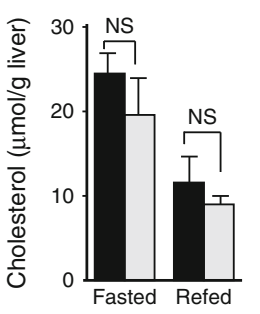

$f$
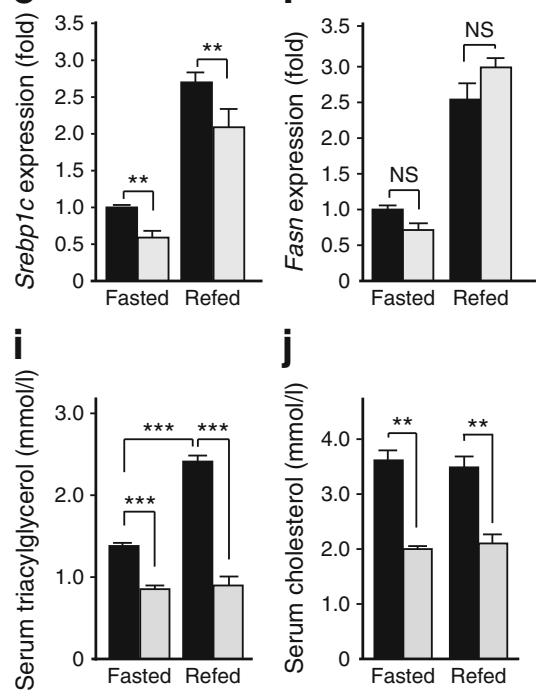

C

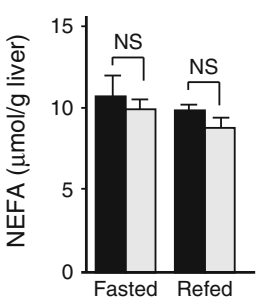

g

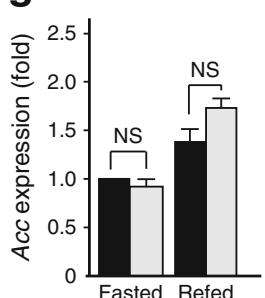

k d

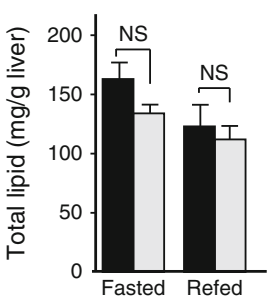

h
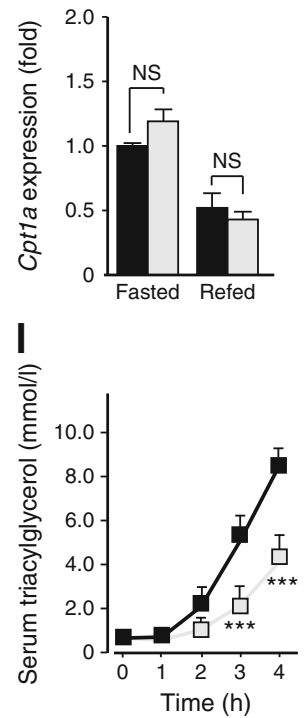

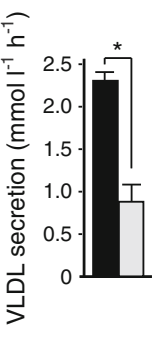

Fig. 2 Lipid profile. (a-d) Triacylglycerol (a), total cholesterol (b), total NEFA (c) and total lipid (d) levels in the livers of Drp/LiKO and control mice under either fasted or refed (for $4 \mathrm{~h}$ ) conditions $(n=6)$. (e-h) Hepatic gene expression of Srebplc (e), Fasn (f), Acc (g) and Cpt1a (h) was determined by real-time quantitative RT-PCR analysis ( $n=5$ per group). Values were normalised to Gapdh and are presented relative to fasting control set at 1. (i-k) Serum concentrations of triacylglycerol (i), total cholesterol (j) and total NEFA (k) in fasted and refed DrplLiKO and control mice $(n=6)$. (l) Serum triacylglycerol levels in control (black squares) and DrplLiKO mice (grey squares) after refeeding for $4 \mathrm{~h}$, measured after tyloxapol injection. The bar graph shows the rate of hepatic triacylglycerol secretion ( $n=5-7$ per group). Data are expressed as means \pm SE. Black bars, control mice; grey bars, DrplLiKO mice. ${ }^{*} p<0.05, * * p<0.01$ and $* * * p<0.001$, calculated by Student's $t$ test (l, triacylglycerol level), two-way ANOVA (a-k) or repeated measures two-way ANOVA (l, VLDL secretion)
Serum triacylglycerol values, reflecting hepatic triacylglycerol secretion, were measured after intraperitoneal tyloxapol injection. DrplLiKO mice showed significantly reduced rates of hepatic triacylglycerol secretion (Fig. 21). Reduced levels of serum triacylglycerol might be attributed to, in part, defective VLDL secretion.

\section{Drp1LiKO mice display improved glucose metabolism} Drp/LiKO mice showed significantly improved glucose tolerance (assessed by an intraperitoneal glucose tolerance test [IPGTT]) compared with control mice (Fig. 3a), while no significant difference was found in plasma insulin levels (Fig. 3b). As a result, the HOMA-IR was markedly lower in DrplLiKO mice (Fig. 3c). Consistent with this, an insulin tolerance test (ITT) revealed high insulin sensitivity in DrplLiKO mice (Fig. 3d). This improved glucose profile was not observed in the NCD-fed mice (ESM Fig. 1 b, c). Analysis of the mRNA expression of genes encoding enzymes involved in glycolysis (glucokinase [Gck], pyruvate kinase $[P k 1]$ ) and gluconeogenesis (glucose 6-phosphatase [G-6-pase, also known as G6pc], phosphoenolpyruvate carboxykinase $1[P c k 1]$, peroxisome proliferator-activated receptor $\gamma$ co-activator $1 \propto[$ Ppargcla]) showed that Gck, G-6-pase and Ppargcla were significantly downregulated in the liver of fasted DrplLiKO mice (Fig. 3e-i).

\section{Loss of Drp1 altered the mitochondria-ER positional} relationship Electron microscopy analysis of mitochondria showed a dispersed pattern in the cytosol of hepatocytes from both control and DrplLiKO mice, while the morphology of Drp 1LiKO mitochondria was swollen (Fig. 4a-d). As shown in Fig. 4a, c, ER morphology in control hepatocytes showed a long tubular network located along mitochondria, and there were relatively equal intervals between mitochondria and ER. In striking contrast, the ER in hepatocytes from DrplLiKO mice was swollen and irregular-shaped, disrupting the relative 

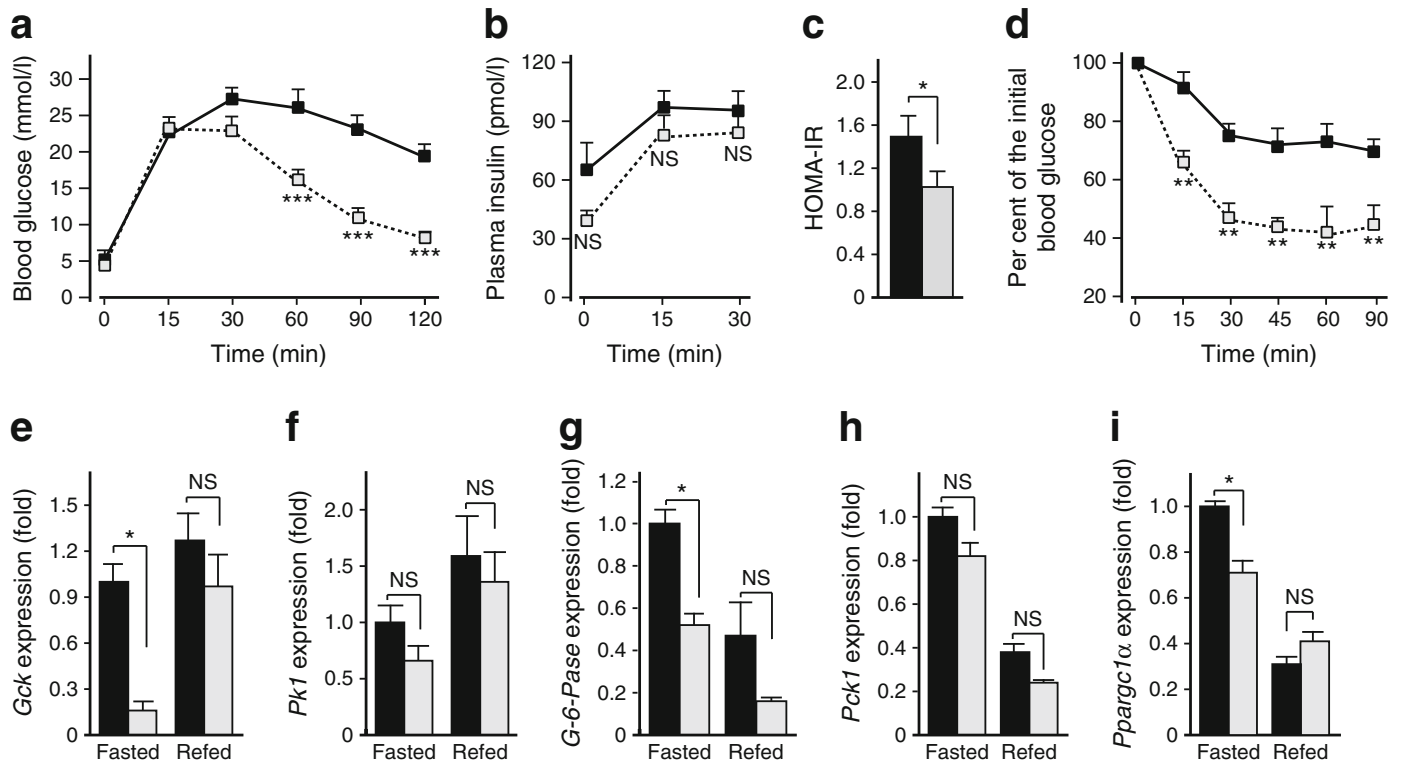

Fig. 3 Amelioration of glucose metabolism in DrplLiKO mice. (a, b) Blood glucose (a) and plasma insulin (b) values during IPGTT of Drp ILiKO and control mice under HFD conditions $(n=11-16)$. (c) HOMA-IR scores in HFD-fed DrplLiKO and control mice $(n=6)$. (d) Blood glucose profile during ITT of Drp /LiKO and control mice $(n=6)$. (e-i) Hepatic expression levels of the genes encoding glucose kinase (Gck) (e), pyruvate kinase ( $P k l)$ (f), glucose-6-phosphatase (G-6-pase) $(\mathrm{g})$, phosphoenolpyruvate carboxykinase $(P c k l)(\mathbf{h})$ and peroxisome

positional relationship between ER and mitochondria (Fig. 4b, d). A change in ER morphology was also evident, although less severe, in the liver of DrplLiKO mice fed the NCD (ESM Fig. 2). Morphometric analysis revealed that the total number of mitochondria in hepatocytes from DrplLiKO mice was significantly reduced but the number of large mitochondria $\left(>10,000 \mu \mathrm{m}^{2}\right)$ was significantly increased (Fig. 4e, f). Despite the morphological change in mitochondria, the expression levels of the mitochondrial import machinery components, TOM20 and TOM40, and the mitochondrial respiratory chain complexes were not changed by loss of Drpl (Fig. 4g, h). Consistent with these observations, the ATP content and mitochondrial respiratory activity were not altered in DrplLiKO mice (Fig. 4i-k).

\section{Loss of Drp1 causes ER stress and activates the eukaryotic} translation factor $2 \alpha$-activating transcription factor 4 FGF21 pathway Liver mRNA from control and DrplLiKO mice was subjected to microarray analysis. Genes were selected using the criterion of a $z$ score of $\geq 2$, which identified 526 upregulated and 640 downregulated genes in Drp 1 LiKO mice. The top five upregulated genes are shown in Table 1 . Among these, P8, Atf3 and Trb3 (also known as Trib3) are known to be ER stress-response genes [17-19], indicating that Drpl defects induce ER stress. Meg3, a maternally expressed imprinted gene, has a function of tumour suppression by proliferator-activated receptor $\gamma$ co-activator $1 \alpha$ (Ppargcla) (i) determined by real-time quantitative RT-PCR analysis ( $n=3-8$ per group). Values were normalised to Gapdh and are presented relative to fasting control set at 1 . Data are expressed as means \pm SE. Black bars and squares, control mice; grey bars and squares, DrplLiKO mice. ${ }^{*} p<0.05$, $* * p<0.01$ and $* * * p<0.001$, calculated by Student's $t$ test (c), two-way ANOVA $(\mathbf{e}-\mathbf{i})$ or repeated measures two-way $\operatorname{ANOVA}(\mathbf{a}, \mathbf{b}, \mathbf{d})$

activation of P53 [20]. Insulin-like growth factor binding protein 1 is potently induced during ER stress by activating transcription factor 4 (ATF4) [21]. Further confirmation by real-time RT-PCR analysis revealed that ER stressresponse genes were significantly increased even in mice on the NCD, and were further increased by the HFD (Fig. 5a-d). Phosphorylation of eukaryotic initiation factor $2(\mathrm{eIF} 2 \alpha)$ is a critical early step in the regulation of protein synthesis during ER stress. Western blotting analysis revealed an increase in phosphorylation of $\operatorname{eIF} 2 \alpha$ in the liver of DrplLiKO mice along with a concomitant increase in its downstream targets ATF4 and C/EBP homologous protein (CHOP) (Fig. 5e). Recently, MAM dysfunction was found to be associated with hepatic insulin resistance [13]. In addition, liver-specific $M f n 2$-knockout mice showed glucose intolerance with hepatic insulin resistance [12]. Therefore, we evaluated the level of phosphorylated Akt (pAkt) in DrplLiKO mouse liver. As shown in Fig. 5f, addition of exogenous insulin increased pAkt levels in both control and Drp/LiKO mouse livers. However, a significant reduction was found in the amount of pAkt after insulin stimulation in the liver of Drp 1 LiKO mice compared with control mice, indicating hepatic insulin resistance in Drp 1 LiKO mice.

FGF21 has recently emerged as a hepatic stress hormone and an effective metabolic regulator of glucose and lipid 

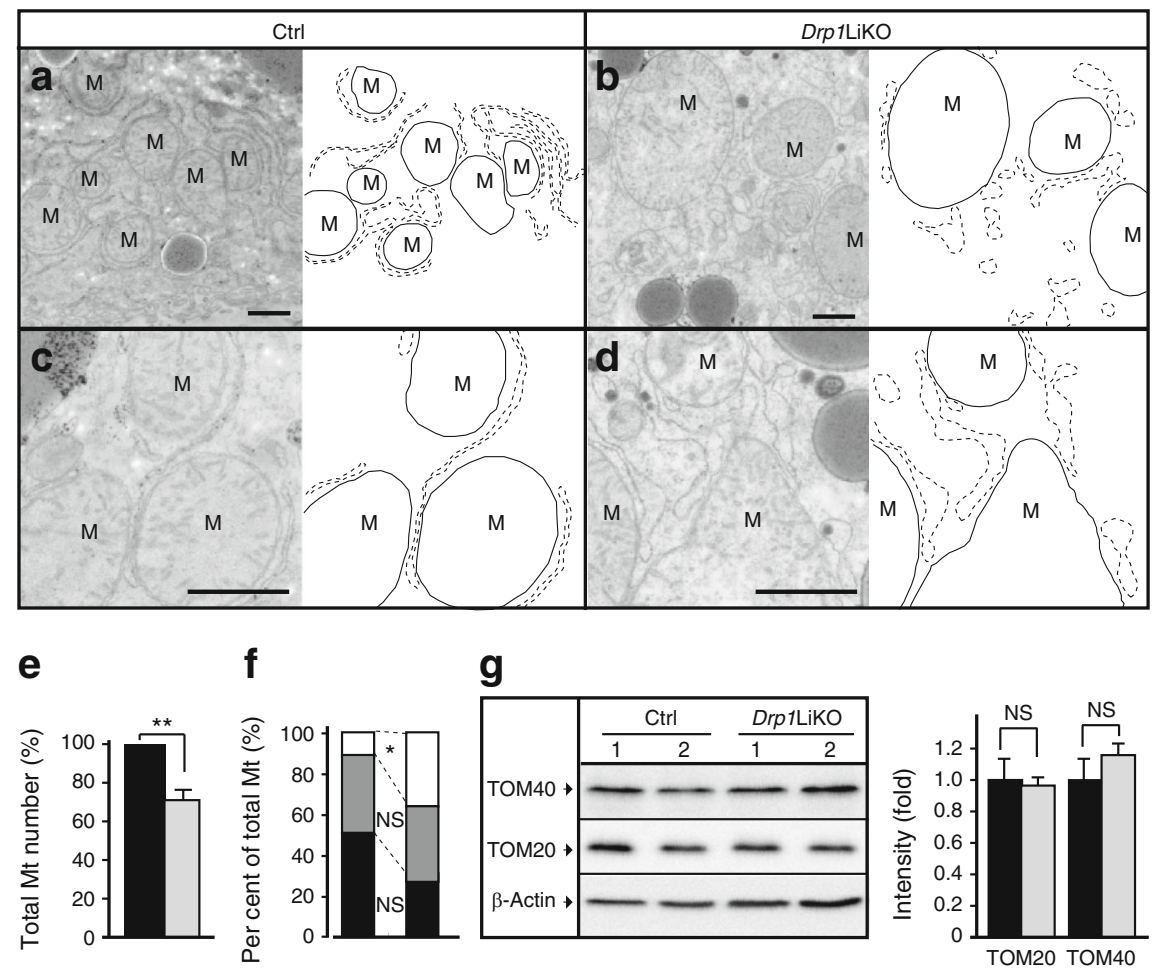

h
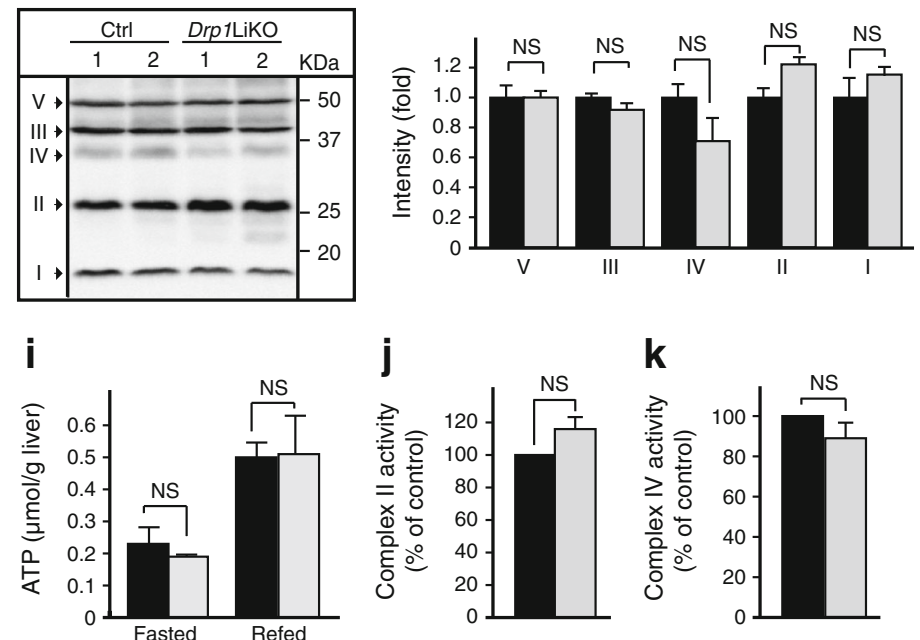

Fig. 4 Altered positional relationship between mitochondria and ER in hepatocytes of DrplLiKO mice on HFD. (a-d) Electron micrographs of livers from control (Ctrl) and Drp /LiKO mice on HFD at magnification $\times 3,000(\mathbf{a}, \mathbf{b})$ and $\times 7,000(\mathbf{c}, \mathbf{d})$. Traces of ER and mitochondrial profiles are also shown. M, mitochondria; dotted lines, ER outline. Scale bar, $1 \mu \mathrm{m}$. (e) Total mitochondria (Mt) number in 24 graphs in DrplLiKO mice is presented as the percentage of control mice. (f) Distribution of mitochondrial areas $<5,000 \mu \mathrm{m}^{2}$ (black bars), 5,000-10,000 $\mathrm{\mu m}^{2}$ (grey bars) or $>10,000 \mu \mathrm{m}^{2}$ (white bars) is shown as a percentage of mitochondria measured. Control, $n=439$; DrplLiKO, $n=289$. (g) Western blots representative of two mice showing the expression of mitochondrial membrane proteins, TOM20 and TOM40. The bar graph shows

homeostasis [22-24]. Furthermore, Fgf21 expression has shown to be induced by the protein kinase R-like ER kinase (PERK)-eIF2 $\alpha$-ATF4 axis [25-27]. Activation of the elF2 $\alpha-$ intensities of protein bands, quantified by densitometric scanning. (h) Western blots representative of two mice showing the expression of each subunit of mitochondrial respiratory complex. The bar graph shows intensities of protein bands, quantified by densitometric scanning. Values were normalised to $\beta$-actin and are presented relative to control set at 1 . (i) Hepatic ATP content in DrplLiKO and control mice under either fasted or refed conditions $(n=6)$. $(\mathbf{j}, \mathbf{k})$ Mitochondrial respiratory rates of complex II (j) and complex IV (k) are shown by oxygen consumption in control and Drp 1LiKO mice liver mitochondria. Data are expressed as means \pm SE. Black bars, control mice; grey bars, Drp1LiKO mice. ${ }^{*} p<0.05$ and ${ }^{* *} p<0.01$, calculated by Student's $t$ test $(\mathbf{e}-\mathbf{h}, \mathbf{j}, \mathbf{k})$ or two-way ANOVA (i)

ATF4 axis was demonstrated in the liver of DrplLiKO mice (Fig. 5e) so we examined $F g f 21$ gene expression in DrplLiKO mice. When mice were fed with NCD, no 
Table 1 Top upregulated genes in Drp/LiKO mouse liver

\begin{tabular}{|c|c|c|c|c|c|c|}
\hline Gene & Description & Control signal & KO signal & $z$ score & Ratio & GenBank accession \\
\hline Atf3 & Activating transcription factor 3 & 89.21 & $4,816.02$ & 11.19 & 53.99 & NM_007498 \\
\hline Nuprl (P8) & Nuclear protein 1 & 202.36 & $8,763.69$ & 10.57 & 43.31 & NM_019738 \\
\hline Meg3 & Maternally expressed 3 & 48.12 & $4,218.52$ & 10.28 & 87.67 & NM_027652 \\
\hline Igfbpl & Insulin-like growth factor binding protein 1 & $5,310.81$ & $87,869.71$ & 9.98 & 16.55 & NM_008341 \\
\hline Trb3 (Trib3) & Tribbles homolog 3 & $1,445.83$ & $29,318.34$ & 8.55 & 20.28 & NM_175093 \\
\hline
\end{tabular}

statistical difference in Fgf21 mRNA expression was found between control and DrplLiKO mice (Fig. 5g). Feeding with HFD resulted in a significant increase in Fgf2 1 mRNA expression in Drp 1 LiKO mice but not in control mice (Fig. 5g). Serum FGF21 concentrations in Drp1LiKO mice were significantly higher than those in control mice on NCD (Fig. 5h); these levels were increased further by an HFD burden. As shown in Fig. 5i, j, mRNA expression of the receptor complex for $F g f 21$, Fgfrl and $K l b$, was increased in WAT of Drp $1 \mathrm{LiKO}$ mice, suggesting that FGF21 has a possible feedforward effect on its own signalling pathway [28]. The expression of FGF21 was further assessed by immunohistochemistry. There was scattered and faint staining in control liver (Fig. 5k). In contrast, a remarkable increase in FGF21 protein was observed in the cytoplasm of hepatocytes from DrplLiKO mouse livers (Fig. 5k).

Increased energy expenditure in Drp1LiKO mice The adipocytes in WAT were smaller in DrplLiKO mice than in control mice (Fig. 6a). The expression of genes involved in lipolysis, such as those encoding hormone sensitive lipase (Lipe) and adipose triacylglycerol lipase (Atgl, also known as Pnpla2), was significantly increased in adipose tissue of DrplLiKO mice (Fig. 6b, c). The expression of the gene encoding adiponectin (Adipoq) was significantly increased in Drp1LiKO mice (Fig. 6d). However, no statistical difference was found in serum adiponectin levels when comparing control mice $(40.39 \pm 7.89 \mu \mathrm{g} / \mathrm{ml}, n=9)$ with Drp 1 LiKO mice $(40.01 \pm 7.87 \mu \mathrm{g} / \mathrm{ml}, n=10)(p=0.94)$. Given that we observed no appreciable attenuation of food intake (Fig. 1c), we hypothesised that FGF21 might increase energy expenditure. As measured by indirect calorimetry, oxygen consumption in DrplLiKO mice was significantly increased compared with that in control mice (Fig. 6e). The respiratory exchange ratio (RER) of Drp 1LiKO mice was significantly lower during both light and dark cycles (Fig. 6f), indicating an increased use of lipids in DrplLiKO mice. The effect of FGF21 on energy expenditure is attributed to its regulation of the thermogenic genes in WAT and brown adipose tissue
(BAT) [29]. We therefore investigated the expression of the genes such as Cidea, Pgcla (also known as Ppargcla), Ucpl and Cptlb in adipose tissue. The expression of Pgcla and Ucpl was significantly induced in DrplLiKO BAT (Fig. 6g). The expression of Cidea and Ucpl was significantly increased in DrplLiKO WAT, suggesting browning of WAT (Fig. 6h). Furthermore, expression of the target genes for FGF21, such as those encoding dual specificity phosphatase 4 (Dusp4), leptin receptor (Lepr), FBJ osteosarcoma oncogene ( $c F O s)$ and early growth response 1 (Egrl), have been analysed [30-32]. We found that Dusp4 and Lepr mRNA expression levels were significantly increased in the WAT of Drp 1LiKO mice, while cFos and Egrl expression levels were not increased (Fig. 6i), consistent with the previous observation that exogenous FGF21 failed to increase the expression of immediate early genes such as cFos and Egrl in obese mice [32].

\section{Discussion}

In this study, we showed that lack of DRP1 in mouse hepatocytes caused ER stress, which was dramatically exacerbated by an HFD. In addition, we found that the PERK-eIF2 $\alpha$-ATF4 pathway was dramatically activated in the liver of Drp 1LiKO mice (Fig. 5a-e). Loss of DRP1 in the liver had no effect on the inositol-requiring $1 \alpha$ (IRE $1 \alpha$ ) and ATF6 pathways (data not shown). Although ATF6, PERK and IRE1 $\alpha$ share functionally similar luminal sensing domains and are activated in cells treated with ER stress inducers in vitro, they are selectively activated and regulate diverse downstream target genes in vivo [33]. Our results suggest that mitochondrial fission may act upstream to the cascade of the ER stress response and that communication between mitochondria and the ER is indispensable to the maintenance of cellular homeostasis. In this study, we provided direct evidence that loss of DRP1 alters ER architecture. Mitochondrial dynamics, which are changed in response to metabolic burden, may play an important role in maintaining ER morphology and function. 
We observed that a defect in mitochondrial fission and subsequent ER stress promoted the expression of FGF21 in the liver and that the increased FGF21 in turn functions as a metabolic regulator, exhibiting anti-obesity and anti-diabetes effects [22-24]. A recent report by Kim et al demonstrated that autophagy deficiency in muscle leads to protection against obesity and insulin resistance by inducing FGF21 [34].
Interestingly, autophagy deficiency also results in morphologically abnormal, swollen mitochondria, reminiscent of those found in the liver of Drp/LiKO mice on an HFD. These results led us to hypothesise that defects in the inter-organelle communication between mitochondria and ER might depend on FGF21 signalling as a self-defence system to maintain metabolic homeostasis. Recently, Sebastian et al reported that
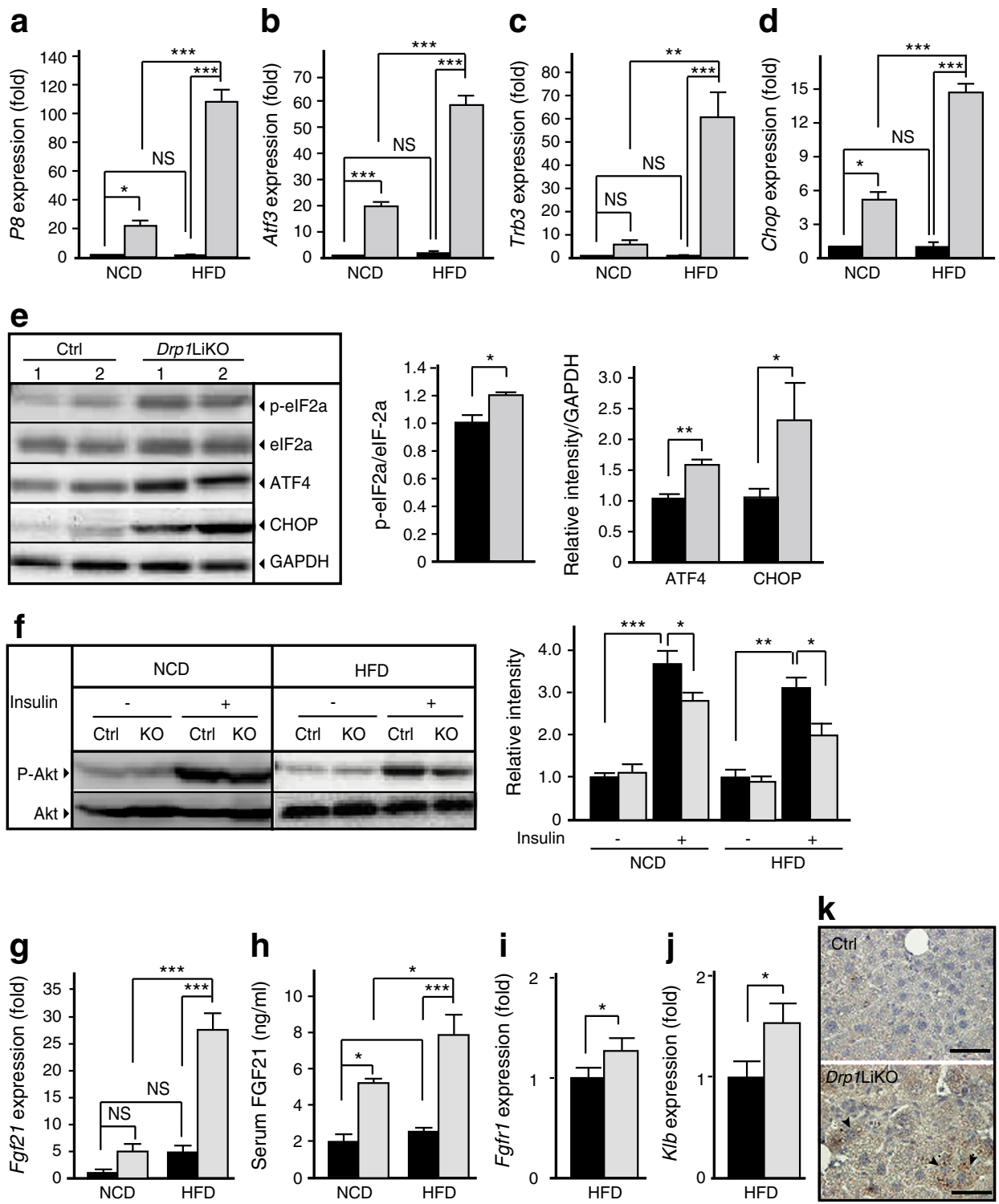

Fig. 5 Increased ER stress and FGF21 expression in DrplLiKO mouse liver. (a-d) Expression of the genes involved in ER stress. The expression levels of $P 8$ (a), Atf3 (b), Trb3 (c) and Chop (also known as Ddit3) (d) were determined by real-time quantitative RT-PCR analysis of total RNA from liver samples taken from NCD- and HFD-fed mice ( $n=4$ per group). (e) Western blots representative of two mice showing the expression of phosphorylated eIF $2 \alpha$, total eIF $2 \alpha$, ATF4, CHOP and GAPDH. Bar graphs show intensities of protein bands, quantified by densitometric scanning. (f) The phosphorylation levels of Akt (pAkt) in the livers of control and Drp/LiKO mice fed either NCD or HFD. Bar graph shows intensities of protein bands, quantified by densitometric scanning. (g) The

hepatic gene expression of Fgf21 in control and DrplLiKO mice fed either NCD or HFD ( $n=5$ or 6$)$. (h) Serum concentration of FGF21 in control and Drp/LiKO mice under the fasted condition $(n=3-8)$. (i, j) The expression of Fgfrl (i) and $K l b(\mathbf{j})$ in WAT of control and DrplLiKO mice $(n=8)$. (k) Immunohistochemical analyses of FGF21 protein in liver sections from control (Ctrl) and DrplLiKO mice. Arrowheads show concentrated cytoplasmic FGF21. Scale bar, $50 \mu \mathrm{m}$. Black bars, control mice; grey bars, Drp 1 LiKO mice. Data are expressed as means \pm SE. ${ }^{*} p<0.05$, ${ }^{* *} p<0.01$ and $* * * p<0.001$, as calculated by Student's $t$ test $(\mathbf{e}, \mathbf{i}, \mathbf{j})$ or two-way ANOVA $(\mathbf{a}-\mathbf{d}, \mathbf{f}-\mathbf{h})$ 
Fig. 6 Increased energy expenditure in DrplLiKO mice. (a) HE staining of WAT from 20 week-old control (Ctrl) and DrplLiKO mice on HFD. Scale bar, $50 \mu \mathrm{m}$. Adipocyte size was measured and presented relative to control set at 1 (bar graph). $(\mathbf{b}, \mathbf{c})$ The expression of genes involved in lipolysis, Lipe (b) and Atgl (c), in WAT. (d) The expression of adiponectin in WAT. (e, f) Oxygen consumption (e) and RER (f) in 16-week-old mice on HFD. $(\mathbf{g}, \mathbf{h})$ The thermogenic gene expression in BAT (g) and WAT (h) $(n=5)$.

(i) The expression of the target genes for FGF21 in WAT $(n=5)$. For mRNA expression, values were normalised to Gapdh and are presented relative to control set at 1. Black bars, control mice; grey bars, DrplLiKO mice. Data are expressed as means \pm SE. ${ }^{*} p<0.05$, calculated by Student's $t$ test
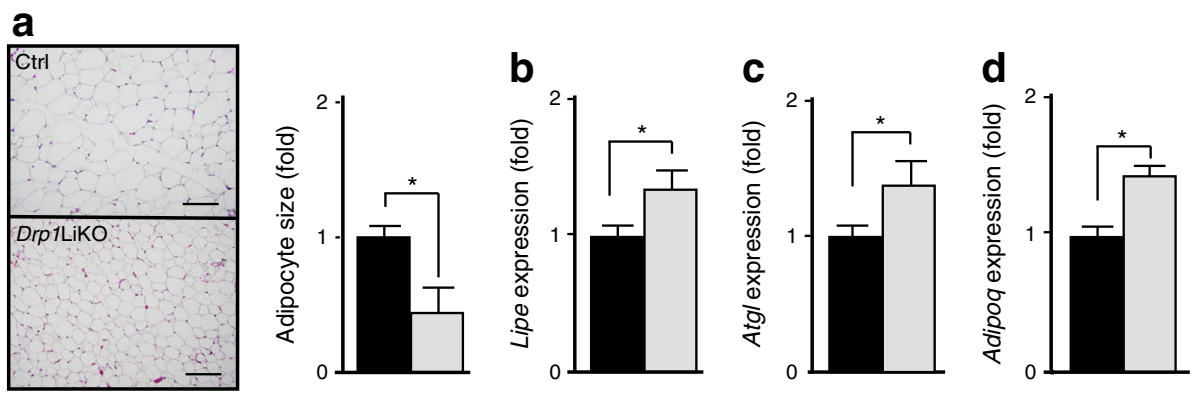
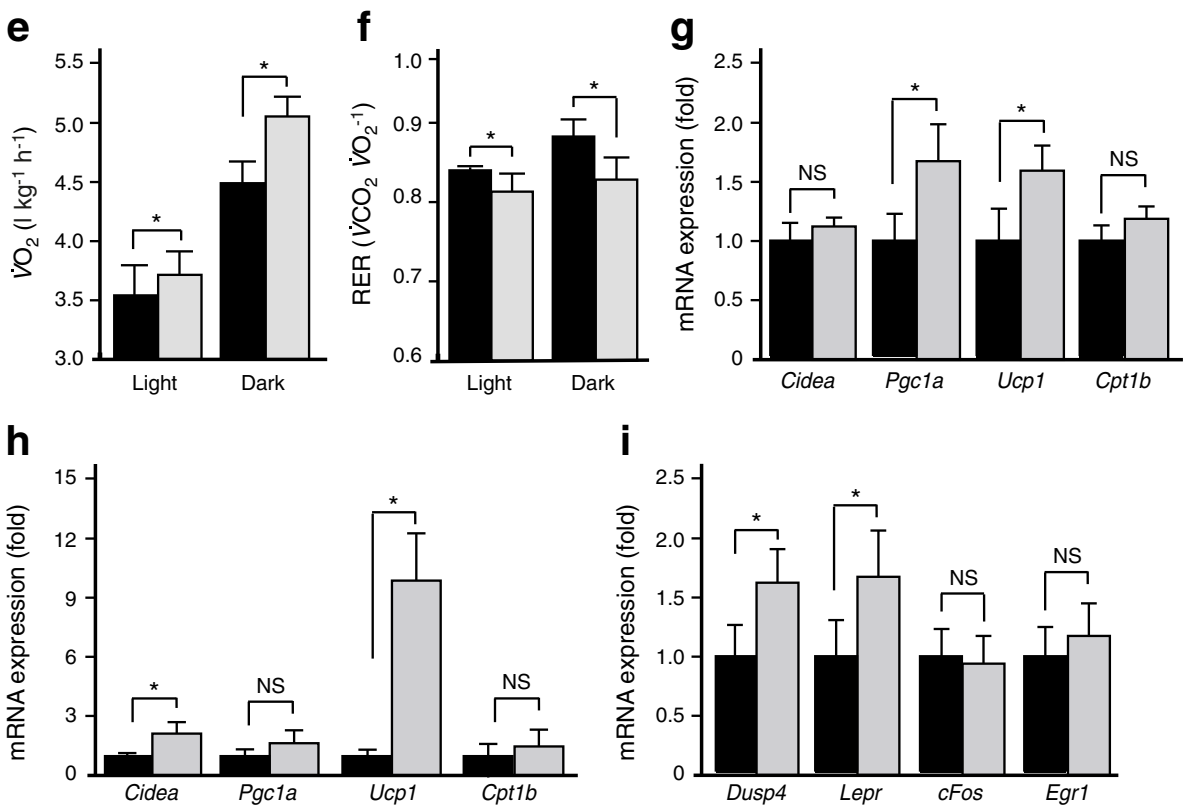

liver-specific $M$ fn2-knockout mice showed glucose intolerance with hepatic insulin resistance [12]. Loss of MFN2 causes impaired mitochondrial respiration, indicating that MFN2 has multiple roles beyond regulating mitochondrial morphogenesis. There may be different roles for mitochondrial fusion and fission in organelle communication inducing Fgf21. FGF21 increases expression of thermogenic genes in adipose tissue and utilisation of lipids. In addition, FGF21 improves insulin sensitivity by increasing glucose uptake in adipocytes [22] and FGF21-deficient mice are insulin resistant and hyperinsulinaemic [35]. It is thus conceivable that the favourable whole-body insulin sensitivity in DrplLiKO mice can be explained, at least in part, by increased FGF21, although they have hepatic insulin resistance.

Energy homeostasis is achieved by multiple mechanisms, including inter-organ communication. As well as humoral factors, recent studies have demonstrated that neuronal pathways play an important role in energy homeostasis as a feedback mechanism, preventing excessive energy intake and enhancing energy expenditure [36]. The interesting possibility that the signal from the liver of DrplLiKO mice uses neuronal pathways in addition to FGF21 cannot be excluded. Nevertheless, our results suggests that mitochondrial dynamics are relevant to understanding the pathogenesis of human metabolic diseases caused by perturbations resulting from overnutrition.

In conclusion, we provide new insight into the role of mitochondrial fission in energy homeostasis. DRP1 may be a potential target for intervention in metabolic diseases such as diabetes and obesity.

Acknowledgements We thank H. Kawate, K. Ashida and K. Ohnaka of the Graduated School of Medical Science, Kyushu University for helpful discussions. We also thank the Research Support Center, Graduate School of Medical Science, Kyushu University, for technical support.

Access to research materials The microarray data from this publication have been submitted to the GEO database http://www.ncbi.nlm.nih. gov/geo/ and assigned the identifier accession: GSE64222.

Funding This work was supported in part by the Japanese Society for the Promotion of Science (JSPS) KAKENHI (MN, Grant no. 26461383, 12F02426; RT, Grant no. 23390247) and Grants-in-Aid for Research Fellowship for Young Science Foundation and Banyu Science Foundation (LW). The work of MN was supported by a grant from the Medical Research Encouragement Prize of The Japan Medical Association.

Duality of interest The authors declare that there is no duality of interest associated with this manuscript. 
Contribution statement $\mathrm{LW}$ and $\mathrm{MN}$ designed the project, analysed the data and wrote the manuscript. LW, TI, DS, YH, YT, SS and SY acquired data. YI and KT contributed to the generation of the mice. All authors contributed to analysis and interpretation of the data, revised the manuscript critically for important intellectual content and approved the final version of the paper to be published. $\mathrm{MN}$ is the guarantor of the article.

\section{References}

1. Karbowski M, Youle RJ (2003) Dynamics of mitochondrial morphology in healthy cells and during apoptosis. Cell Death Differ 10: 870-880

2. Chan D, Frank S, Rojo M (2006) Mitochondrial dynamics in cell life and death. Cell Death Differ 13:680-684

3. Archer SL (2013) Mitochondrial dynamics-mitochondrial fission and fusion in human diseases. N Engl J Med 369:2236-2251

4. Baumann O, Walz B (2001) Endoplasmic reticulum of animal cells and its organization into structural and functional domains. Int Rev Cytol 205:149-214

5. Friedman JR, Lackner LL, West M, DiBenedetto JR, Nunnari J, Voeltz GK (2011) ER tubules mark sites of mitochondrial division. Science 334:358-362

6. Hayashi T, Rizzuto R, Hajnoczky G, Su TP (2009) MAM: more than just a housekeeper. Trends Cell Biol 19:81-88

7. Marchi S, Patergnani S, Pinton P (2014) The endoplasmic reticulum-mitochondria connection: one touch, multiple functions. Biochim Biophys Acta 1837:461-469

8. Otera H, Mihara K (2011) Molecular mechanisms and physiologic function of mitochondrial dynamics. J Biochem 149:241-251

9. Ishihara N, Otera H, Oka T, Mihara K (2013) Regulation and physiologic functions of GTPases in mitochondrial fusion and fission in mammals. Antioxid Redox Signal 19:389-399

10. Shen Q, Yamano K, Head BP et al (2014) Mutations in Fis1 disrupt orderly disposal of defective mitochondria. Mol Biol Cell 25:145159

11. Korobova F, Ramabhadran V, Higgs HN (2013) An actindependent step in mitochondrial fission mediated by the ERassociated formin INF2. Science 339:464-467

12. Sebastian D, Hernandez-Alvarez MI, Segales J et al (2012) Mitofusin 2 (Mfn2) links mitochondrial and endoplasmic reticulum function with insulin signaling and is essential for normal glucose homeostasis. Proc Natl Acad Sci U S A 109:5523-5528

13. Tubbs E, Theurey P, Vial G et al (2014) Mitochondria-associated endoplasmic reticulum membrane (MAM) integrity is required for insulin signaling and is implicated in hepatic insulin resistance. Diabetes 63:3279-3294

14. Ishihara N, Nomura M, Jofuku A et al (2009) Mitochondrial fission factor Drp1 is essential for embryonic development and synapse formation in mice. Nat Cell Biol 11:958-966

15. Folch J, Lees M, Sloane Stanley GH (1957) A simple method for the isolation and purification of total lipides from animal tissues. $\mathrm{J}$ Biol Chem 226:497-509

16. Nomura M, Zhu H-L, Wang L et al (2014) SMAD2 disruption in mouse pancreatic beta cells leads to islet hyperplasia and impaired insulin secretion due to the attenuation of ATP-sensitive $\mathrm{K}+$ channel activity. Diabetologia 57:157-166
17. Carracedo A, Lorente M, Egia A et al (2006) The stress-regulated protein $\mathrm{p} 8$ mediates cannabinoid-induced apoptosis of tumor cells. Cancer Cell 9:301-312

18. Huang J, Viswakarma N, Yu S et al (2011) Progressive endoplasmic reticulum stress contributes to hepatocarcinogenesis in fatty acylCoA oxidase 1-deficient mice. Am J Pathol 179:703-713

19. Sanchez-Lopez E, Zimmerman T, Gomez del Pulgar T, Moyer MP, Lacal Sanjuan JC, Cebrian A (2013) Choline kinase inhibition induces exacerbated endoplasmic reticulum stress and triggers apoptosis via CHOP in cancer cells. Cell Death Dis 28:e933

20. Zhou Y, Zhong Y, Wang Y et al (2007) Activation of $\mathrm{p} 53$ by MEG3 non-coding RNA. J Biol Chem 282:24731-24742

21. Marchand A, Tomkiewicz C, Magne L, Barouki R, Garlatti M (2006) Endoplasmic reticulum stress induction of insulin-like growth factor-binding protein-1 involves ATF4. J Biol Chem 281: 19124-19133

22. Kharitonenkov A, Shiyanova TL, Koester A et al (2005) FGF-21 as a novel metabolic regulator. J Clin Invest 115:1627-1635

23. Coskun T, Bina HA, Schneider MA et al (2008) Fibroblast growth factor 21 corrects obesity in mice. Endocrinology 149:6018-6027

24. Cheung BM, Deng H (2014) Fibroblast growth factor 21: a promising therapeutic target in obesity-related diseases. Expert Rev Cardiovasc Ther 12:659-666

25. Schaap FG, Kremer AE, Lamers WH, Jansen PL, Gaemers IC (2013) Fibroblast growth factor 21 is induced by endoplasmic reticulum stress. Biochimie 95:692-699

26. Kim KH, Jeong YT, Kim SH et al (2013) Metformin-induced inhibition of the mitochondrial respiratory chain increases FGF21 expression via ATF4 activation. Biochem Biophys Res Commun 440: $76-81$

27. Kim SH, Kim KH, Kim H-K et al (2015) Fibroblast growth factor 21 participates in adaptation to endoplasmic reticulum stress and attenuates obesity-induced hepatic metabolic stress. Diabetologia 58:809-818

28. Keipert S, Ost M, Johann K et al (2014) Skeletal muscle mitochondrial uncoupling drives endocrine cross-talk through the induction of FGF21 as a myokine. Am J Physiol Endocrinol Metab 306: E469-E482

29. Fisher FM, Kleiner S, Douris N et al (2012) FGF21 regulates PGC$1 \alpha$ and browning of white adipose tissues in adaptive thermogenesis. Genes Dev 26:271-281

30. Muise ES, Souza S, Chi A et al (2013) Downstream signaling pathways in mouse adipose tissues following acute in vivo administration of fibroblast growth factor 21. PLoS One 8:e73011

31. Adams AC, Cheng CC, Coskun T, Kharitonenkov A (2012) FGF21 requires $\beta$ klotho to act in vivo. PLoS One 7:e49977

32. Fisher FM, Chui PC, Antonellis PJ et al (2010) Obesity is a fibroblast growth factor 21 (FGF21)-resistant state. Diabetes 59:27812789

33. Cao SS, Kaufman RJ (2012) Unfolded protein response. Curr Biol 22:R622-R626

34. Kim KH, Jeong YT, Oh H et al (2013) Autophagy deficiency leads to protection from obesity and insulin resistance by inducing Fgf21 as a mitokine. Nat Med 19:83-92

35. So WY, Cheng Q, Xu A, Lam KSL, Leung PS (2015) Loss of fibroblast growth factor 21 action induces insulin resistance, pancreatic islet hyperplasia and dysfunction in mice. Cell Death Dis 6 : e1707

36. Katagiri H, Yamada T, Oka Y (2007) Adiposity and cardiovascular disorders: disturbance of the regulatory system consisting of humoral and neuronal signals. Circ Res 101:27-39 